\title{
5-grass pollen tablets achieve disease control in patients with seasonal allergic rhinitis unresponsive to drugs: a real-life study
}

\author{
Elide Anna Pastorello' \\ Laura Losappio' \\ Stefania Milani² \\ Giuseppina Manzotti ${ }^{3}$ \\ Valentina Fanelli ${ }^{4}$ \\ Valerio Pravettoni ${ }^{5}$ \\ Fabio Agostinis ${ }^{6}$ \\ Alberto Flores D'Arcais ${ }^{7}$ \\ Ilaria Dell'Albani ${ }^{8}$ \\ Paola Puccinelli9 \\ Cristoforo Incorvaia ${ }^{10}$ \\ Franco Frati $^{8}$
}

'Allergy and Immunology Department, Niguarda Hospital, Milan, ${ }^{2}$ Allergy Department, San Marco General Hospital, Bergamo, ${ }^{3}$ Allergy Department, Treviglio Hospital, Bergamo, ${ }^{4}$ Allergy Department, Italian Institute for Auxology, Milan, ${ }^{5}$ Clinical Allergy and Immunology Unit, Foundation IRCCS Ca' Granda Ospedale Maggiore Policlinico, Milan, ${ }^{6}$ Department of Pediatrics, Riuniti Hospital, Bergamo, ${ }^{7}$ Department of Pediatrics, Legnano Hospital, Milan, ${ }^{8}$ Medical and Scientific Department, Stallergenes Italy, Milan, ${ }^{9}$ Regulatory Department, Stallergenes Italy, Milan, ${ }^{10}$ Allergy/Pulmonary Rehabilitation, ICP Hospital, Milan, Italy

Correspondence: Franco Frati Medical and Scientific Department, Stallergenes Italy, Viale Certosa 2, Milan 20155, Italy

$\mathrm{Tel}+3902334541$

Fax +3902 70058778

Email ffrati@stallergenes.it
This article was published in the following Dove Press journal:

Journal of Asthma and Allergy

3 December 2013

Number of times this article has been viewed

\begin{abstract}
Background: An important subpopulation in allergic rhinitis is represented by patients with severe form of disease that is not responsive to drug treatment. It has been reported that grass pollen subcutaneous immunotherapy is effective in drug-resistant patients. In a real-life study, we evaluated the efficacy of 5-grass pollen tablets in patients with grass pollen-induced allergic rhinitis not responsive to drug therapy.
\end{abstract}

Methods: We carried out this multicenter observational study in adults and adolescents with grass-induced allergic rhinitis not responsive to drug therapy who were treated for a year with 5-grass pollen tablets. Clinical data collected before and after sublingual immunotherapy (SLIT) included Allergic Rhinitis and its Impact on Asthma (ARIA) classification of allergic rhinitis, response to therapy, and patient satisfaction.

Results: Forty-seven patients entered the study. By ARIA classification, three patients had moderate to severe intermittent allergic rhinitis, ten had mild persistent allergic rhinitis, and 34 had moderate to severe persistent allergic rhinitis. There were no cases of mild intermittent allergic rhinitis before SLIT. After SLIT, 33 patients had mild intermittent allergic rhinitis, none had moderate to severe intermittent allergic rhinitis, seven had mild persistent allergic rhinitis, and seven had moderate to severe persistent allergic rhinitis. The mean medication score decreased from $4.2 \pm 1.3$ before to $2.4 \pm 2.0$ after SLIT $(P<0.01)$, representing a reduction of $42 \%$. The response to treatment before SLIT was judged as poor by $70 \%$ of patients and very poor by $30 \%$. Patient satisfaction was significantly increased after SLIT $(P<0.01)$.

Conclusion: In real life, most patients with grass pollen-induced allergic rhinitis not responsive to drug treatment can achieve control of the condition with one season of treatment using 5-grass pollen tablets.

Keywords: grass pollen, rhinitis, drug resistance, allergen immunotherapy, effectiveness, patient satisfaction

\section{Introduction}

Allergen immunotherapy (AIT) is based on administration of increasing doses of the causal allergen to reduce clinical reactivity in allergic patients, and therefore is the only treatment targeting the cause and not merely the symptoms of respiratory allergy. ${ }^{1}$ The outcome is achieved via the immunologic mechanism of action of AIT, that mostly consists of restoring a Th1-dominant T-cell response and generating specific T-suppressor cells. ${ }^{2}$ These effects are clinically expressed by tolerance to the administered allergen, resulting, as far as respiratory allergy is concerned, in a decreased burden of rhinitis and asthma. ${ }^{3}$ AIT may be administered via the injected route (subcutaneous immunotherapy, SCIT) or the sublingual route (sublingual immunotherapy, SLIT). The efficacy of SLIT in allergic rhinitis and asthma is demonstrated 
by a number of meta-analyses, ${ }^{4-10}$ including subpopulations such as children, ${ }^{5,7}$ patients specifically evaluated for asthma, ${ }^{6}$ and patients treated with particular allergens such as the house dust mite ${ }^{9}$ or grass pollen. ${ }^{10}$ Another important subpopulation is represented by patients inadequately controlled on drug treatment, who have severe symptoms during the pollen season and consequently a high burden of disease and high economic costs. ${ }^{11}$

Frew et al were the first to show that one season of SCIT was significantly more effective than placebo with regard to symptoms and medication scores in patients with grass pollen-induced rhinoconjunctivitis who had had an unsatisfactory response to standard drug treatment. ${ }^{12}$ In their study, two maintenance doses of 10,000 and 100,000 standardized quality (SQ) units were used, with higher efficacy but also lower tolerability using the higher dose. It is well known that SLIT is significantly better tolerated than SCIT, but no trials are available showing that SLIT is effective in patients not adequately controlled by drugs. A recent post hoc analysis of published studies using 5 -grass pollen tablets showed that better clinical efficacy was achieved by immunotherapy in patients with more severe allergic rhinitis, although it was not reported if these patients were unresponsive to drugs. ${ }^{13}$ In a real-life study, we evaluated the efficacy of SLIT using 5 -grass pollen tablets in patients poorly responsive to drug treatment.

\section{Materials and methods \\ Patients}

Seven allergy centers in Italy, namely Niguarda Hospital (Milan), San Marco General Hospital (Bergamo), Treviglio Hospital (Bergamo), Italian Institute for Auxology (Milan), Maggiore General Hospital (Milan), Riuniti Hospital (Bergamo), and Legnano Hospital (Milan) participated in this study. Male and female subjects aged 12-45 years with seasonal allergic rhinitis were included. For inclusion, patients had to have had symptoms of rhinitis during the grass pollen season (from mid April to end of June) for at least 2 consecutive years, an unsatisfactory response to drug treatment in the previous season, as assessed by the patients themselves using a specific questionnaire, and a positive skin prick test with grass pollen extracts. Allergic rhinitis was classified according to Allergic Rhinitis and its Impact on Asthma (ARIA) guidelines. ${ }^{3}$ The main exclusion criteria were allergic rhinoconjunctivitis caused by cosensitization and likely to influence symptoms during the study, previous AIT, and the usual contraindications to AIT. ${ }^{14}$

\section{Study design}

This multicenter observational study, known as ORS (Oralair ${ }^{\circledR}$ Rhinitis Solution, Stallergenes, Antony, France) was conducted in two phases: at the end of the 2010 pollen season, the investigators identified patients with allergic rhinitis unresponsive to drug therapy (retrospective phase); and during the 2011 pollen season collected clinical data from these patients, who were treated preseasonally and coseasonally with 5 -grass pollen tablets (prospective phase). Medication scores in the two seasons were calculated by attributing a score of 3 to topical steroids, a score of 2 to oral antihistamines or antileukotrienes, and a score of 1 to topical antihistamines or nasal decongestants.

The primary objective of the study was to investigate the effectiveness of first-year SLIT treatment in patients with allergic rhinitis unresponsive to drug treatment. The secondary objectives were to assess use of rescue medication, the presence of comorbidities, and patient satisfaction, measured using a visual analog scale that has been proven to be a valid tool in evaluating allergic rhinitis, ${ }^{15}$ in particular for patient satisfaction. ${ }^{16}$ Using the visual analog scale, patients assessed their level of satisfaction by indicating a position along a continuous line between two points from 0 (very dissatisfied) to 10 (very satisfied).

For data collection, we used a paper case report form recording the ARIA classification of allergic rhinitis, ${ }^{3}$ onset of symptoms, clinically relevant allergen(s), comorbidities, response to therapy, and patient satisfaction.

Written informed consent was obtained before entering the study, and in the case of minors was obtained from next of kin, caregivers, or guardians. The study was conducted in accordance with good clinical practice guidelines. The ethics committees at each of the participating sites approved the study, ie, the ethics committees of Niguarda Hospital (Milan), San Marco General Hospital (Bergamo), Treviglio Hospital (Bergamo), Italian Institute for Auxology (Milan), Maggiore General Hospital (Milan), Riuniti Hospital (Bergamo), and Legnano Hospital (Milan).

\section{Immunotherapy}

SLIT was performed using a preparation of 5 -grass pollen in tablets (Oralair ${ }^{\circledR}$; Stallergenes S.A., Antony, France), the characteristics of which have already been reported. ${ }^{17}$ Briefly, the preparation contains a mixture of Phleum pratense, Dactylis glomerata, Anthoxanthum odoratum, Lolium perenne, and Poa pratensis, covering the immunoglobulin E sensitization profiles of grass-allergic patients in the Mediterranean area. ${ }^{18}$ Each tablet was taken sublingually once daily in the 
morning before breakfast and was kept under the tongue until complete dissolution before swallowing. It was administered according to a pre-coseasonal schedule of SLIT, ie, started 4 months before the expected start of the grass pollen season and stopped at the end of the pollen season, for an overall duration of 6 months. Any local or systemic adverse effects of SLIT were to be recorded by the patients.

\section{Statistical analysis}

A central organization (IBIS Informatica, Milan, Italy) undertook the data entry and statistical analysis. Nominal and discrete parameters were reported as contingency tables (frequency and percentage). McNemar's test was used to compare the two pollen seasons (2010 versus 2011). Continuous parameters were reported as the frequency, mean, standard deviation, median, first and third quartile, and minimum and maximum. Comparison of the two pollen seasons was performed using the Student's $t$-test for paired data, and the difference between seasons was reported with the related $95 \%$ confidence interval. Inferential tests were evaluated at a $5 \%$ level of significance. Statistical analysis was carried out using BMDP Dynamic 2009 version 8.2 software (BMDP Statistical Software, Inc., Los Angeles, CA, USA).

\section{Results}

Forty-seven patients of mean age 25.7 years were included in the study, comprising 23 males and 24 females, with 38 adults and nine adolescents. Allergic rhinitis had been present for $12 \pm 8.5$ years in the adults and for $5.5 \pm 4.2$ years in the adolescents. Of the 47 patients, at inclusion in 2010, none $(0 \%)$ reported mild intermittent allergic rhinitis, three $(6.4 \%)$ had moderate to severe intermittent allergic rhinitis, ten $(21.3 \%)$ had mild persistent allergic rhinitis, and $34(72.3 \%)$ had moderate to severe persistent allergic rhinitis. The response to symptomatic treatment was judged as poor by 33 patients $(70.2 \%)$ and very poor by 14 patients (29.8\%). During the 2011 grass pollen season, after treatment with 5 -grass pollen tablets, 33 patients $(79.2 \%)$ reported mild intermittent allergic rhinitis, none $(0 \%)$ had moderate to severe intermittent allergic rhinitis, seven (14.9\%) had mild persistent allergic rhinitis, and seven (14.9\%) had moderate to severe persistent allergic rhinitis.

Comparing the two seasons, 31 patients classified as having mild or moderate to severe persistent disease when treated with symptomatic drugs changed to mild intermittent allergic rhinitis when treated with 5 -grass pollen tablets $(P<0.01)$. Figure 1 shows the changes in ARIA classification for the two seasons. All patients were sensitized to grass pollen. Among the adolescents, six were monosensitized and three were also sensitized to the house dust mite. Adults showed a number of other sensitizations, ie, house dust mite in 11 patients, olive pollen in seven, birch pollen in six, cypress pollen in four, Parietaria pollen in four, and ragweed pollen in three patients.

Table 1 shows the comorbidities reported by patients, the most common being conjunctivitis and asthma. The mean medication score changed from $4.2 \pm 1.3$ before to $2.4 \pm 2.0$

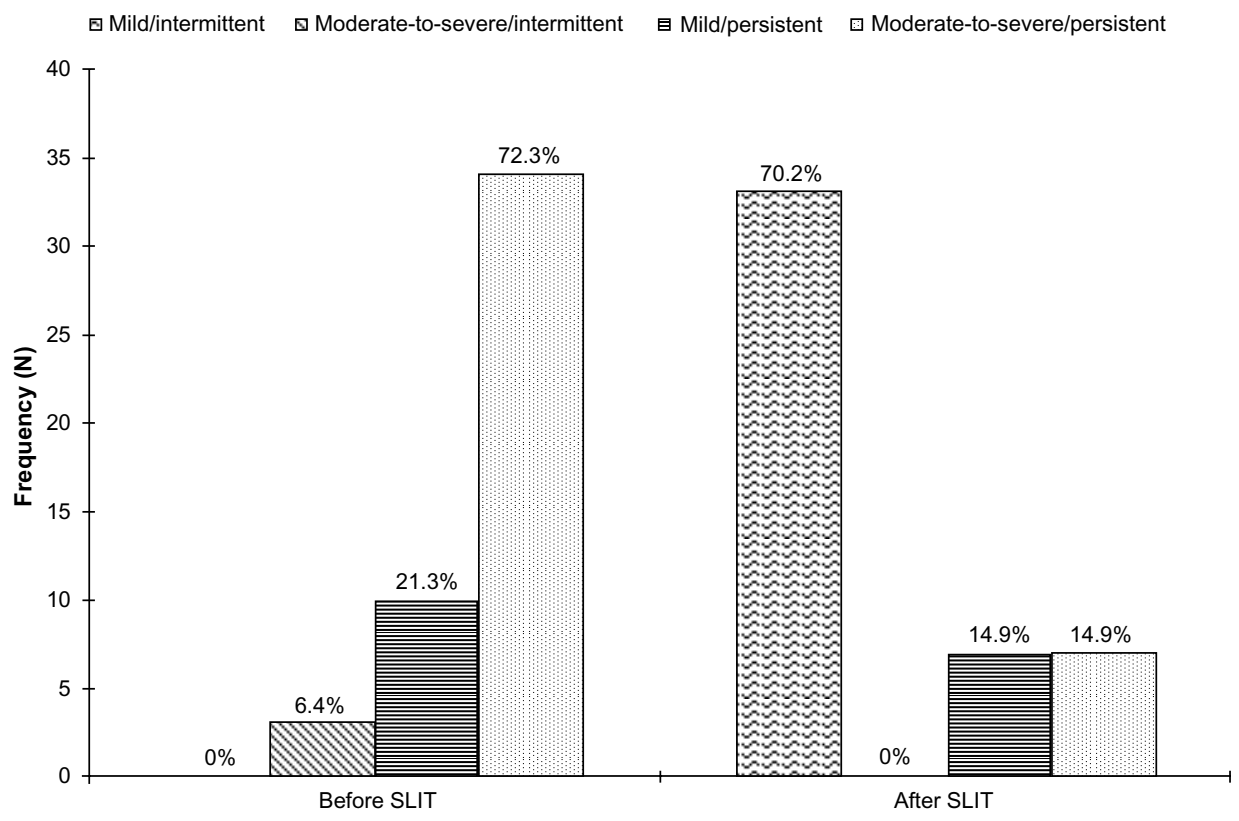

Figure I ARIA classification of allergic rhinitis before and after treatment with 5-grass pollen tablets. Abbreviations: ARIA, Allergic Rhinitis and its Impact on Asthma; SLIT, sublingual immunotherapy. 
Table I Assessment of comorbidities in study population

\begin{tabular}{|c|c|c|c|}
\hline \multirow[t]{2}{*}{ Comorbidity } & $\begin{array}{l}\text { Adults } \\
(n=38)\end{array}$ & $\begin{array}{l}\text { Adolescents } \\
(n=9)\end{array}$ & $\begin{array}{l}\text { Total } \\
(n=47)\end{array}$ \\
\hline & n (\%) & n (\%) & n (\%) \\
\hline Sinusitis & $0(0.0)$ & $0(0.0)$ & $0(0.0)$ \\
\hline Conjunctivitis & $26(68.4)$ & $8(88.9)$ & $34(72.3)$ \\
\hline Asthma & $19(50.0)$ & $0(0.0)$ & $19(40.4)$ \\
\hline Turbinate hypertrophy & $2(5.3)$ & $0(0.0)$ & $2(4.3)$ \\
\hline Polyposis & $0(0.0)$ & $0(0.0)$ & $0(0.0)$ \\
\hline Sleep disturbance & $0(0.0)$ & $0(0.0)$ & $0(0.0)$ \\
\hline Others & $4(10.5)$ & $0(0.0)$ & $4(8.5)$ \\
\hline
\end{tabular}

after SLIT $(P<0.01)$, representing a decrease of $42 \%$ (Figure 2). Twelve patients did not use any rescue medication during treatment with SLIT. In particular, a significant decrease was found in the use of oral antihistamines (46.8\%) and nasal decongestants (21.3\%). Also, concerning the consumption of drugs for asthma, a decrease, albeit not significant, was found, corresponding to $6.4 \%$ for both $\beta 2$-agonists and inhaled corticosteroids. The mean visual analog score for patient satisfaction increased from $3 \pm 1.3$ before to $8.7 \pm 1.3$ after SLIT, indicating a significant improvement $(P<0.01$, Figure 3).

\section{Discussion}

Patients with severe allergic rhinitis warrant particular attention. A definition of severe chronic upper airway disease has been introduced to identify those patients whose symptoms are inadequately controlled despite adequate pharmacologic treatment based on guidelines, and thus have impaired quality of life, social functioning, sleep, and school/ work performance. ${ }^{19} \mathrm{~A}$ recent reappraisal of the current status of knowledge on severe chronic upper airway disease listed a need for " ... defining success of medical treatment including immunotherapy in terms of control in allergic rhinitis". ${ }^{20}$ However, thus far, only one study has addressed this issue. In a large study of patients with grass pollen-induced allergic rhinitis not controlled by standard drug treatment, 203 were randomized to maintenance with 100,000 SQ units, 104 to 10,000 SQ units, and 103 to placebo, with both active treatments being significantly more efficacious than placebo. The 100,000 SQ unit regimen was more effective, but the 10,000 SQ unit regimen caused fewer side effects. ${ }^{12}$ It is surprising that no other study has been done in this area, either with SCIT or SLIT, that has the advantage of being much better tolerated. ${ }^{21}$ In addition, SLIT meets the requirements for use as treatment for pollen-allergic patients because it has the highest evidence of efficacy as demonstrated by meta-analyses ${ }^{4-8,10}$ and offers benefit over SCIT in terms of compliance and pharmacoeconomic aspects. ${ }^{22}$

In a real-life study, we sought to assess the effectiveness of SLIT in the form of the 5-grass pollen preparation in drugresistant patients, given that this therapy has already been demonstrated to be effective and safe in both adults ${ }^{17}$ and children. ${ }^{23}$ The 5 -grass pollen tablets were prescribed after

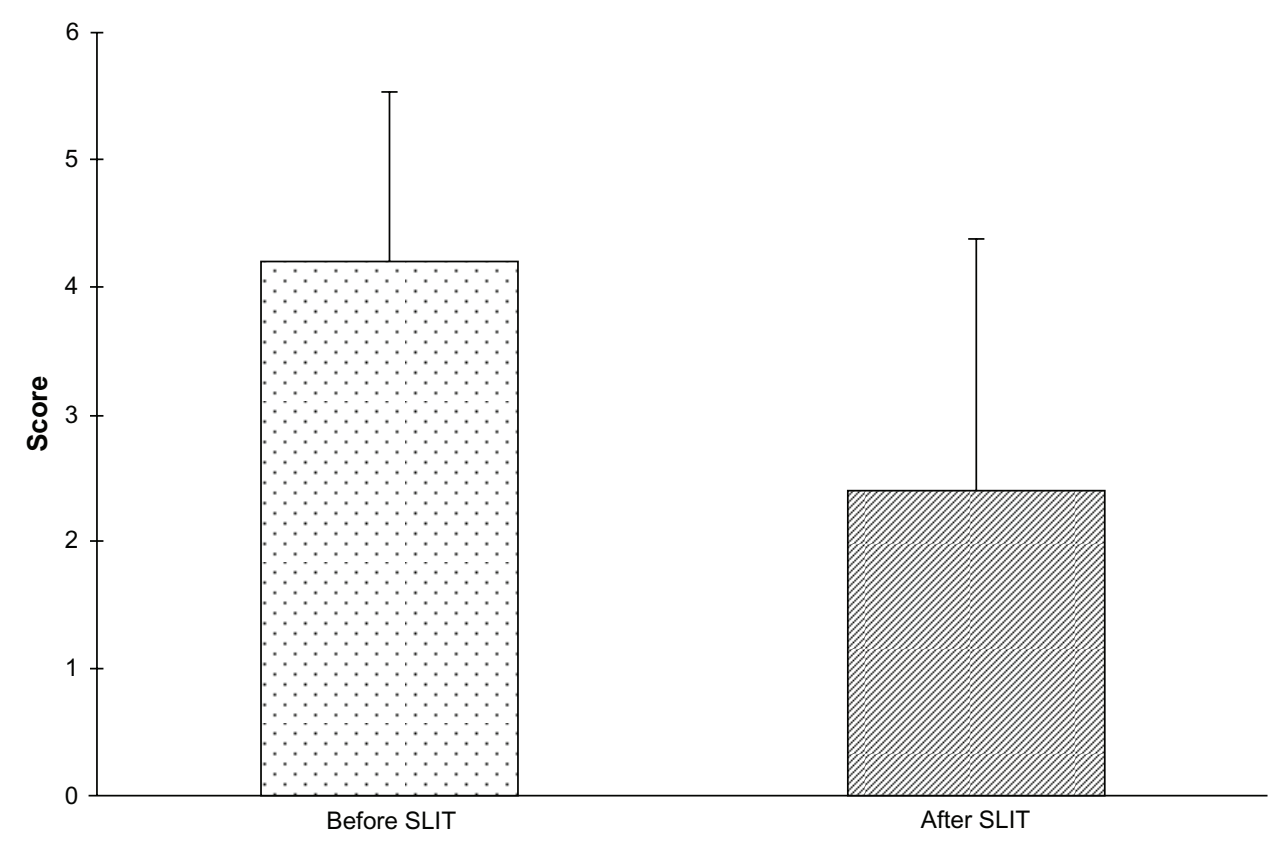

Figure 2 Medication score before and after treatment with 5 -grass pollen tablets. The medication score in the 2011 pollen season was significantly lower than in the 2010 pollen season (mean difference $-1.8,95 \%$ confidence interval -2.1 to $-1.5, P<0.01$ ).

Abbreviation: SLIT, sublingual immunotherapy. 


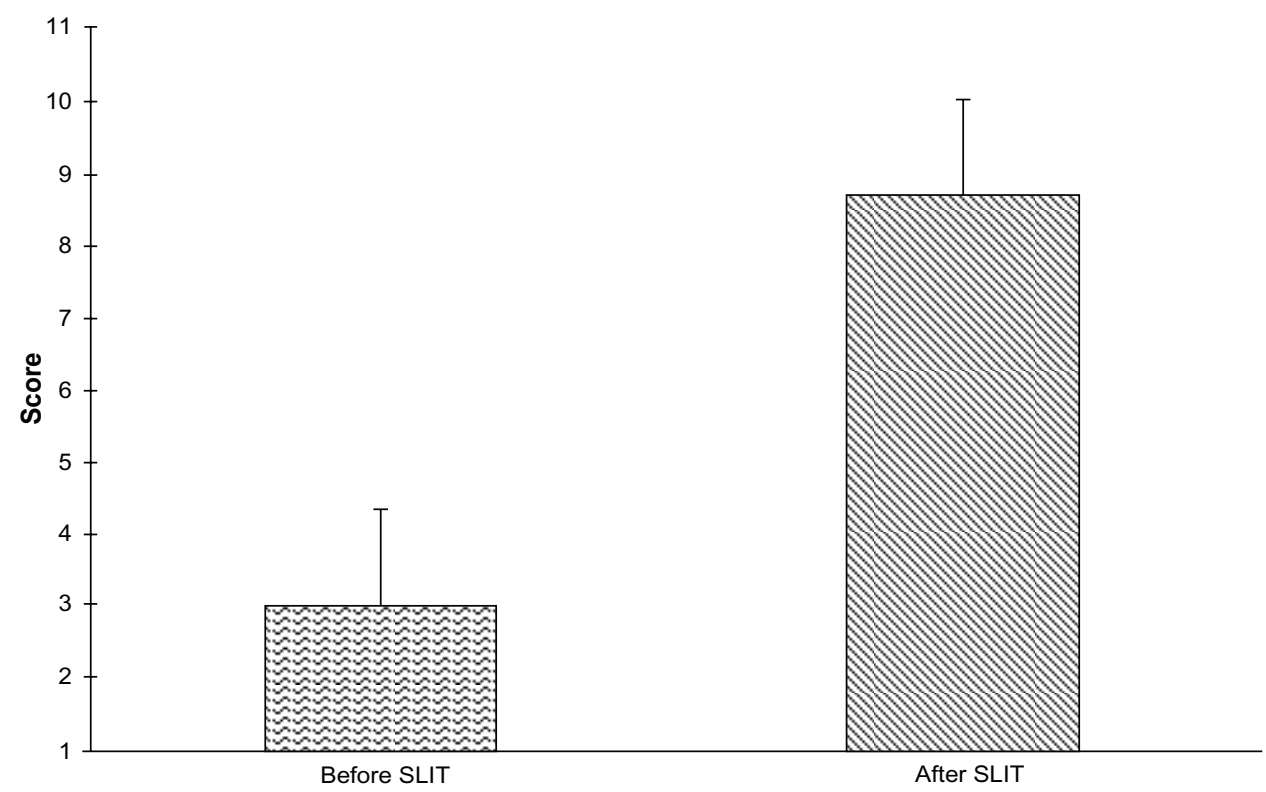

Figure 3 Patient satisfaction before and after treatment with 5-grass pollen tablets. Patient satisfaction in $201 \mathrm{I}$ pollen season was significantly higher than in the 2010 pollen season (mean difference $-5.72,95 \%$ confidence interval -6.33 to $-5.12, P<0.01$ ).

Abbreviation: SLIT, sublingual immunotherapy.

a pollen season in which standard drug treatment, ie, antihistamines, nasal corticosteroids, and nasal decongestants, had been ineffective. Following 5-grass pollen therapy, the clinical stage of allergic rhinitis, as defined by ARIA classification, was overturned. The number of patients with mild intermittent allergic rhinitis increased from 0 to 33 , while the number of patients with moderate to severe persistent allergic rhinitis decreased from 34 to 7 , ie, the severity of symptoms was reversed. Mean medication scores decreased significantly from $4.2 \pm 1.3$ before to $2.4 \pm 2.0$ after SLIT. This finding indicates that 5-grass pollen tablets are able to achieve clinical control of allergic rhinitis in patients unresponsive to drugs, as previously demonstrated for SCIT. ${ }^{12}$ Asthma was not a major issue for our patients, but a decrease in use of bronchodilators and inhaled corticosteroids was found with SLIT. This is not unexpected, given the strong relationship between allergic rhinitis and asthma. ${ }^{3}$ As far as factors related to failure of antiallergic drugs are concerned, a recent paper on severe chronic upper airway disease suggested a number of disease-related, patient-related, and treatment-related factors. ${ }^{20}$ Among them, comorbidities, such as chronic sinusitis, and adherence to medical treatment seem particularly important. We can exclude a role for comorbidity in our population, because no patient reported sinusitis. On the other hand, it is well known that adherence is a major problem in treating allergic rhinitis. ${ }^{24,25}$

The patients in our study reported having been adherent with their prescribed drug treatment, but no stringent method of control, such as counting the assumed drug doses or frequent phone calls, was used. In any case, it is unlikely that patients with symptoms unresponsive to drugs, ie, the type of population we targeted, use less than the optimal dose recommended by their physicians. In fact, in a recent Italian survey of 1,379 patients with allergic rhinitis undertaken by 107 general practitioners, adherence with ARIA guidelines was evaluated according to severity classification, and adherence was observed to increase according to the severity of the condition. In particular, adherence was satisfactory in patients with moderate to severe persistent allergic rhinitis, with a rate of $89 \%$ for those with allergic rhinitis alone and $95 \%$ for those with allergic rhinitis and asthma. The authors concluded that adherence to ARIA guidelines is satisfactory only in patients with more severe disease. ${ }^{26}$

It is of particular interest to focus on the relevance of drug resistance in patients with allergic rhinitis. To the best of our knowledge, only one study that included an epidemiologic assessment is available, and showed that 111 (13.9\%) of 796 patients had uncontrolled allergic rhinitis after treatment. The evaluation included a number of parameters, including total symptom scores for rhinitis, a visual analog scale, the Rhinoconjunctivitis Quality of Life Questionnaire, and the Allergy-specific Work Productivity and Impairment Questionnaire. A cluster-randomized trial model was used to mimic real life, with no exclusion of patients at randomization, so as to include the broad range of patients regularly seen by physicians. ${ }^{27}$ 
Concerning patient satisfaction with treatment, it is reasonable to assume that this is related to clinical outcome. Recent studies have investigated patient satisfaction with treatment for allergic rhinitis and allergic asthma. In a survey conducted in 21 allergy centers in Italy, only $33.5 \%$ of patients with allergic rhinitis and $40.7 \%$ of patients with asthma were satisfied with their symptomatic treatment. The main factors associated with treatment dissatisfaction were female gender, comorbidity, and the severity of symptoms of allergic rhinitis and asthma. ${ }^{28}$ Similar results were reported in a survey of more than 5,000 patients in Europe, with one third being dissatisfied with the treatment prescribed. ${ }^{29}$ In our study, all patients were dissatisfied with their medical treatment because nonresponsiveness to drugs was an inclusion criterion. However, the level of satisfaction, as measured by the visual analog scale, was very low with drugs but increased significantly with SLIT.

\section{Conclusion}

In the present real-life study, we found that most patients with grass pollen-induced allergic rhinitis who did not respond to drug treatment achieved control of their allergic rhinitis using pre-coseasonal treatment with 5-grass pollen tablets during a one-year period. This finding now needs to be confirmed by randomized controlled trials targeting this important subpopulation of patients with allergic rhinitis.

\section{Disclosure}

FF, IDA, and PP are employees of Stallergenes Italy. CI is a scientific consultant for Stallergenes Italy. The other authors have no conflicts of interest that are directly relevant to the content of this study.

\section{References}

1. Calderon MA, Casale TB, Togias A, Bousquet J, Durham SR, Demoly P. Allergen-specific immunotherapy for respiratory allergies: from meta-analysis to registration and beyond. J Allergy Clin Immunol. 2011;127(1):30-38.

2. Jutel M, Akdis CA. Immunological mechanisms of allergen-specific immunotherapy. Allergy. 2011;66(6):725-732.

3. Bousquet J, Khaltaev N, Cruz AA, et al. Allergic Rhinitis and its Impact on Asthma (ARIA) 2008 update (in collaboration with the World Health Organization, GA(2)LEN and AllerGen). Allergy. 2008;63 Suppl 86: $8-160$.

4. Wilson DR, Torres-Lima M, Durham S. Sublingual immunotherapy for allergic rhinitis: systematic review and meta-analysis. Allergy. 2005;60(1):4-12.

5. Olaguibel JM, Alvarez Puebla MJ. Efficacy of sublingual allergen vaccination for respiratory allergy in children. Conclusions from one meta-analysis. J Investig Allergol Clin Immunol. 2005;15(1):9-16.

6. Penagos M, Passalacqua G, Compalati E, et al. Meta-analysis of the efficacy of sublingual immunotherapy in the treatment of allergic asthma in pediatric patients, 3 to 18 years of age. Chest. 2008;133(3): 599-609.
7. Penagos M, Compalati E, Tarantini F, et al. Efficacy of sublingual immunotherapy in the treatment of allergic rhinitis in pediatric patients 3-18 years of age: a meta analysis of randomized placebo-controlled, double blind trials. Ann Allergy Asthma Immunol. 2006;97(2): 141-148.

8. Radulovic S, Calderon MA, Wilson D, Durham S. Sublingual immunotherapy for allergic rhinitis. Cochrane Database Syst Rev. 2010;12:CD002893.

9. Compalati E, Passalacqua G, Bonini M, Canonica GW. The efficacy of sublingual immunotherapy for house dust mites respiratory allergy: results of a GA2LEN meta-analysis. Allergy. 2009;64(11): $1570-1579$.

10. Di Bona D, Plaia A, Scafidi V, Leto-Barone MS, Di Lorenzo G. Efficacy of sublingual immunotherapy with grass allergens for seasonal allergic rhinitis: a systematic review and meta-analysis. J Allergy Clin Immunol. 2010;126(3):558-566.

11. Ruggeri M, Oradei M, Frati F, et al. Economic evaluation of 5-grass pollen tablets versus placebo in the treatment of allergic rhinitis in adults. Clin Drug Investig. 2013;33(5):343-349.

12. Frew AJ, Powell RJ, Corrigan CJ, Durham SR; UK Immunotherapy Study Group. Efficacy and safety of specific immunotherapy with SQ allergen extract in treatment-resistant seasonal allergic rhinoconjunctivitis. J Allergy Clin Immunol. 2006;117(2):319-325.

13. Howarth P, Malling HJ, Molimard M, Devillier P. Analysis of allergen immunotherapy studies shows increased clinical efficacy in highly symptomatic patients. Allergy. 2012;67(3):321-327.

14. Bousquet J, Lockey R, Malling HJ. Allergen immunotherapy: therapeutic vaccines for allergic diseases. A WHO position paper. J Allergy Clin Immunol. 1998;102(4 Pt 1):558-562.

15. Demoly P, Bousquet PJ, Mesbah K, Bousquet J, Devillier P. Visual analogue scale in patients treated for allergic rhinitis: an observational prospective study in primary care, asthma and rhinitis. Clin Exp Allergy. 2013;43(8):881-888.

16. Baiardini I, Puggioni F, Menoni S, et al. Patient knowledge, perceptions, expectations and satisfaction on allergen-specific immunotherapy: a survey. Respir Med. 2013;107(3):361-367.

17. Didier A, Malling HJ, Worm M, et al. Optimal dose, efficacy, and safety of once daily sublingual immunotherapy with a 5 -grass pollen tablet for seasonal allergic rhinitis. J Allergy Clin Immunol. 2007;120(6): $1338-1345$.

18. Marcucci F, Sensi L, Di Cara G, et al. Which allergen extract for grass pollen immunotherapy? An in vitro study. Immunol Invest. 2010;39(6): 635-644.

19. Bousquet J, Bachert C, Canonica GW, et al. Unmet needs in severe chronic upper airway disease (SCUAD). J Allergy Clin Immunol. 2009;124(3):428-433.

20. Hellings PW, Fokkens WJ, Akdis C, et al. Uncontrolled allergic rhinitis and chronic rhinosinusitis: where do we stand today? Allergy. 2013;68(1):1-7.

21. Gidaro GB, Marcucci F, Sensi L, Incorvaia C, Frati F, Ciprandi G. The safety of sublingual-swallow immunotherapy: an analysis of published studies. Clin Exp Allergy. 2005;35(5):565-571.

22. Incorvaia C, Masieri S, Scurati S, Soffia S, Puccinelli P, Frati F. The current role of sublingual immunotherapy in the treatment of allergic rhinitis in adults and children. J Asthma Allergy. 2011;4:13-17.

23. Wahn U, Tabar A, Kuna P, et al; SLIT Study Group. Efficacy and safety of 5-grass pollen sublingual immunotherapy tablets in pediatric allergic rhinoconjunctivitis. J Allergy Clin Immunol. 2009;123(1):160-166.

24. Maurer M, Zuberbier T. Undertreatment of rhinitis symptoms in Europe: findings from a cross-sectional questionnaire survey. Allergy. 2007;62(9):1057-1063.

25. Bukstein D, Luskin AT, Farrar JR. The reality of adherence to rhinitis treatment: identifying and overcoming the barriers. Allergy Asthma Proc. 2011;32(4):265-271.

26. Maio S, Simoni M, Baldacci S, et al. The ARGA study with Italian general practitioners: prescriptions for allergic rhinitis and adherence to ARIA guidelines. Curr Med Res Opin. 2012;28(10):1743-1751. 
27. Bousquet PJ, Bachert C, Canonica GW, et al. Uncontrolled allergic rhinitis during treatment and its impact on quality of life: a cluster randomized trial. J Allergy Clin Immunol. 2010;126(3):666-668.

28. Ciprandi G, Incorvaia C, Scurati S, et al. Patient-related factors in rhinitis and asthma: the satisfaction with allergy treatment survey. Curr Med Res Opin. 2011;27(5):1005-1011.
29. Chivato T, Valovirta E, Dahl R, et al. Allergy, living and learning: diagnosis and treatment of allergic respiratory diseases in Europe. J Investig Allergol Clin Immunol. 2012;22(3):168-179.

\section{Publish your work in this journal}

The Journal of Asthma and Allergy is an international, peer-reviewed open-access journal publishing original research, reports, editorials and commentaries on the following topics: Asthma; Pulmonary physiology; Asthma related clinical health; Clinical immunology and the immunological basis of disease; Pharmacological interventions and

\section{Dovepress}

new therapies. Issues of patient safety and quality of care will also be considered. The manuscript management system is completely online and includes a very quick and fair peer-review system, which is all easy to use. Visit http://www.dovepress.com/testimonials.php to read real quotes from published authors.

Submit your manuscript here: http://www.dovepress.com/journal-of-asthma-and-allergy-journal 